\title{
Rasgos diagnósticos en frutos y residuos secos de la cocción de chañar (Geoffroea decorticans, Fabaceae) para identificar prácticas poscolecta
}

\author{
Valentina Saur Palmieri, Cecilia Trillo y M. Laura López \\ Recibido 31 de enero 2019. Aceptado 17 de mayo 2019
}

\begin{abstract}
RESUMEN
El chañar es un árbol de frutos comestibles de gran importancia cultural en distintas comunidades de Argentina. En el norte de la provincia de Córdoba, su consumo es notable y forma parte del conocimiento ecológico tradicional que poseen los pobladores del sector. A partir de información etnobotánica-etnoarqueológica obtenida previamente, en el presente trabajo se registraron las formas de cocción actuales de chañar en la localidad de Cerro Colorado, a fin de estudiar las transformaciones experimentadas por los frutos procesados y los residuos emanados de diferentes técnicas culinarias. Luego de caracterizar histológicamente esta drupa, se determinaron los rasgos diagnósticos adquiridos por las muestras secas luego de las prácticas poscolecta de lavado, hervido, amasado y colado, así como del consumo directo. Esta información constituye la base para la investigación paleoetnobotánica sobre las actividades ejercidas en el pasado a los macrorrestos de chañar recuperados arqueológicamente. Se pretende contribuir así al estudio de los cambios y continuidades en el consumo de frutos nativos comestibles en el período prehispánico y la actualidad.
\end{abstract}

Palabras clave: Cerro Colorado; Etnobotánica; Norte de Córdoba; Paleoetnobotánica; Frutos silvestres comestibles.

\begin{abstract}
DIAGNOSTIC TRAITS OF FRUIT AND THE DRIED RESIDUES OF CHAÑAR (GEOFFROEA DECORTICANS, FABACEAE) COOKING USED TO IDENTIFY POST-HARVESTING PRACTICES. The chañar tree is an edible fruited species that has strong cultural importance for several Argentinean communities. In the north of Córdoba province, consumption of this fruit is significant, and is part of the Traditional Ecological Knowledge of the region's inhabitants. On the basis of previous ethnobotanical-ethnoarchaeological research, contemporary chañar cooking methods in the town of Cerro Colorado were recorded in order to study the transformations undergone by processed fruit and the residues generated by different cooking techniques. After histological characterization of this drupe, diagnostic traits acquired by dry samples after post-harvest practices such as washing, boiling, kneading, and straining, as well as direct consumption, were determined. Results establish a basis for paleoethnobotanical investigation into activities performed in the past related to archaeologically recovered chañar macro remains. The objective is to contribute to the study of changes and continuities in the use of native edible fruits from the pre-Hispanic period to the present.
\end{abstract}

Keywords: Cerro Colorado; Ethnobotany; North of Córdoba; Paleoethnobotany; Wild edible fruits.

\footnotetext{
Valentina Saur Palmieri. Consejo Nacional de Investigaciones Científicas y Técnicas (CONICET). Facultad de Ciencias Exactas, Físicas y Naturales, Universidad Nacional de Córdoba (FCEFyN-UNC). Av. Vélez Sarsfield 299 (5000), Córdoba, Argentina. E-mail: altea345@hotmail.com

Cecilia Trillo. Instituto Multidisciplinario de Biología Vegetal (IMBIV). FCEFyN-UNC. Av. Vélez Sarsfield 299 (5000), Córdoba, Argentina. E-mail: ceciliatrillol@gmail.com

María Laura López. Consejo Nacional de Investigaciones Científicas y Técnicas (CONICET). Facultad de Ciencias Naturales y Museo, Universidad Nacional de La Plata (FCNyM-UNLP). Av. 60 y 122 (1900), Unidad Anexa, Laboratorio I29, La Plata, Buenos Aires, Argentina. E-mail: mllopezdepaoli@gmail.com
} 


\section{INTRODUCCIÓN}

Los estudios arqueobotánicos en Argentina, y en el resto del mundo, han comenzado a avanzar más allá de la simple identificación del recurso vegetal recuperado en diversos sitios y contextos arqueológicos, y en la actualidad afrontan la determinación de prácticas sociales en las cuales las plantas estuvieron involucradas. Por tal, indagar en estudios etnobotánicos que proveían información esencial sobre dichas prácticas comenzó a ser necesario, no solo para dar cuenta de la posible manipulación de frutos y semillas, sino también para contar con detalles precisos sobre las improntas que cada práctica de procesamiento ha dejado en el vegetal. Este tipo de estudio, el cual puede ser englobado dentro de la etnobotánica-etnoarqueológica (sensu Lema 2009), fue propuesto por Hillman durante los años 1970-1980 (Hillman 1984). En su trabajo, este investigador estableció la importancia de determinar qué restos van surgiendo de las diferentes etapas de la producción de trigo en Turquía, para detectar qué potenciales vestigios vegetales pueden hallarse en diferentes contextos arqueológicos. Realizó descripciones morfológicas precisas de las plantas estudiadas, que permitieron reconocer la fragmentación durante el procesamiento (Hillman y Davis 1990). En los últimos años, Capparelli y colegas (Capparelli y Lema 2010; Capparelli et al. 2011 y los trabajos allí citados) han recalcado la magnitud de llevar a cabo este tipo de estudios. En Argentina, son emblemáticas las investigaciones desarrolladas por Capparelli (2007, 2008, 2011; Capparelli y Lema 2011) sobre Prosopis en Hualfín y su aplicabilidad en macrorrestos del sitio El Shincal de Quimivil (Catamarca). Es interesante remarcar que la cercanía entre pueblo actual y sitio arqueológico es la situación ideal para la realización de este tipo de estudios etnobotánicos-etnoarqueológicos (Margaritis y Jones 2006), debido a que las prácticas de procesamiento actuales pueden exhibir una cierta continuidad del conocimiento ecológico tradicional. Aunque este es transmitido generacionalmente, no debe desconocerse el dinamismo que lleva implícito (Pochettino y Lema 2008), por ejemplo, mediante la incorporación de otros saberes. No obstante, los resultados "puros" -es decir, solo los referidos a los atributos que cada técnica genera en el material vegetal- son válidos para su aplicabilidad en regiones alejadas. Así, Capparelli y Prates (2015) reconocieron prácticas de procesamiento sobre restos de Prosopis en la Patagonia a partir de los datos extraídos en el noroeste argentino.
Las especies silvestres como algarrobos (Prosopis Griseb.), chañar (Geoffroea decorticans (Gillies ex Hook. \& Arn.) Burkart), mistol (Sarcomphalus mistol (Griseb.) Hauenschild), entre otras, constituyen recursos nativos alimenticios propicios para avanzar en la determinación de prácticas presentes y pretéritas. Ampliamente consumidos como alimento en la actualidad, han sido registrados en diversos sitios arqueológicos del país. En esta oportunidad, el presente trabajo se centrará en el reconocimiento de prácticas realizadas con chañar. Este árbol de la familia Fabaceae se distribuye en Perú, Bolivia, Paraguay, Chile, Uruguay y Argentina, donde crece desde el norte hasta la porción septentrional de Patagonia. Posee un fruto indehiscente que corresponde a una drupa ovoide-globosa, de 1,5-3,5 cm de longitud y de 1,5-2,4 cm de latitud. Su epicarpo es anaranjado-rojizo, glabro y liso. Puede presentar pequeñas manchas circulares oscuras (Figura $1 \mathrm{~A}$, D). El mesocarpo es pastoso, abundante y dulce, formado principalmente por disacáridos ( $\approx 30 \%)$. El endocarpo es ovoide-fusiforme, leñoso, rugoso y blanquecino, de 1,5-2 cm de longitud y 1,2-1,5 cm de latitud, y posee un alto contenido lignocelulósico $(97 \%)$. Presenta una semilla con superficie ondulada por los surcos transversales del endocarpo (Figura 1B, C) (Orrabalis et al. 2014; Demaio et al. 2015).

Debido a los numerosos usos que pueden dársele a esta especie (alimenticio, maderable, tintóreo, forrajero, melífero, medicinal, veterinario), representa un recurso muy valorado por distintos pueblos de la actualidad (Figura 2) (Trillo et al. 2014; Demaio et al. 2015; Montani y Scarpa 2016).

Con relación al aspecto alimenticio, los frutos de chañar tienen gran importancia para las comunidades originarias del Gran Chaco del norte argentino, como los chorote, wichi, moqoit y tapiete, que los consumen como fruta fresca o los muelen para elaborar bebidas fermentadas ("aloja") o no fermentadas ("añapa") (Maranta 1987; Arenas 2003; Arenas y Scarpa 2007; Montani y Scarpa 2016; Rosso y Scarpa 2017). Los chorote, tapiete, tobas y wichis también realizan unas "bolas" moldeando la harina con agua o grasa (Maranta 1987; Arenas 2003; Arenas y Scarpa 2007; Montani y Scarpa 2016). Además, las semillas, ricas en lípidos y proteínas (Maestri et al. 2001), son aprovechadas por wichis y tobas (Maranta 1987; Arenas 2003). Los pobladores criollos de la misma región fitogeográfica también hacen uso de estos frutos. En La Rioja, Santiago del Estero, San Luis y Formosa los comen crudos y cocinan arrope (Adaro 1918; Di Lullo 1935; Figueroa 

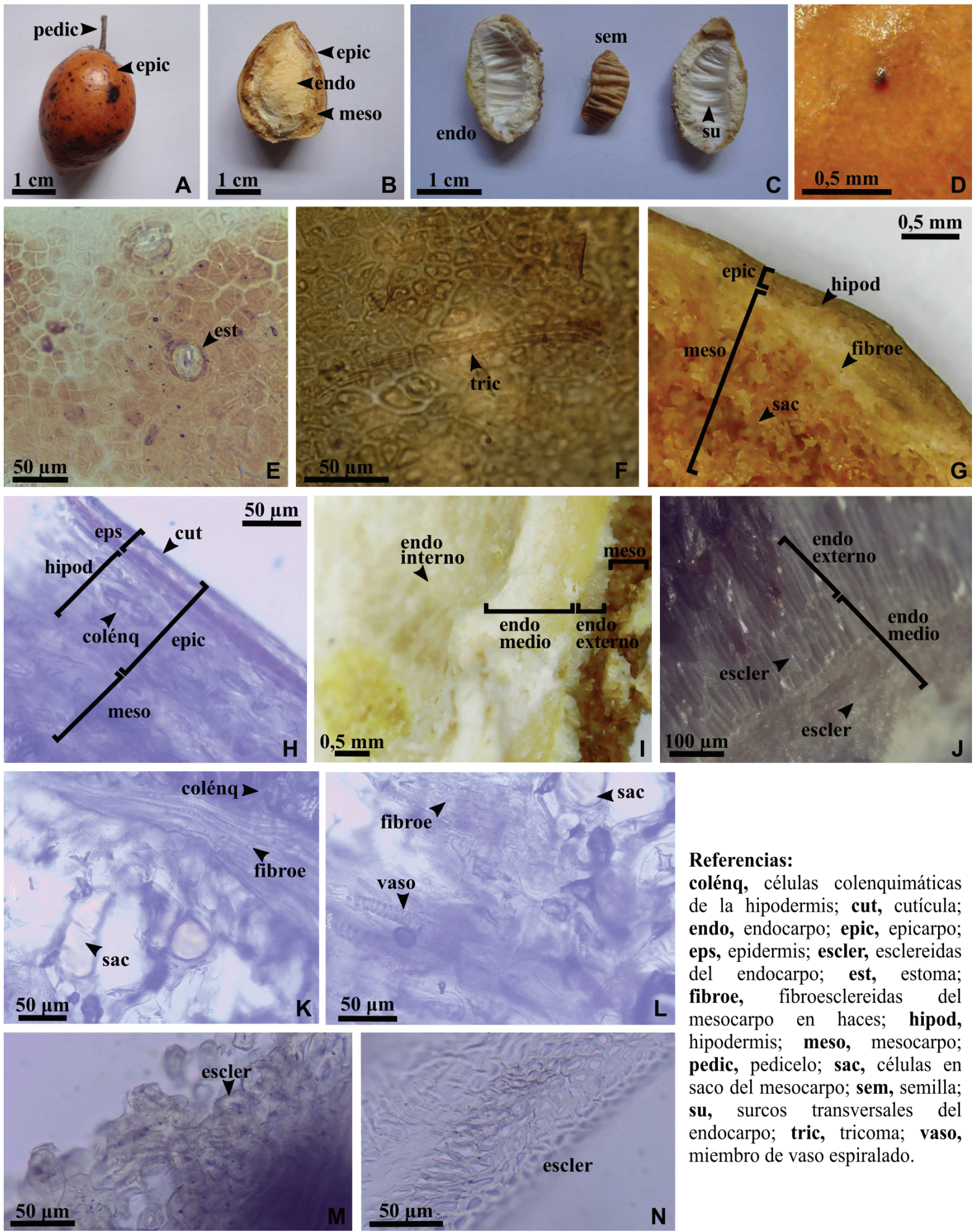

\section{Referencias:}

colénq, células colenquimáticas de la hipodermis; cut, cutícula; endo, endocarpo; epic, epicarpo; eps, epidermis; escler, esclereidas del endocarpo; est, estoma; fibroe, fibroesclereidas del mesocarpo en haces; hipod, hipodermis; meso, mesocarpo; pedic, pedicelo; sac, células en saco del mesocarpo; sem, semilla; su, surcos transversales del endocarpo; tric, tricoma; vaso, miembro de vaso espiralado.

Figura 1. Morfología interna y externa del fruto de chañar. A: vista exterior; B: corte longitudinal; C: vista interna del endocarpo en corte longitudinal por la línea de sutura y semilla. Se aprecia superficie ondulada de esta última; D: epicarpo en vista superficial. Se observa glabro y liso; E y F: epidermis en vista superficial; G y H: capas histológicas externas en corte transversal; I y J: corte transversal por endocarpo; K: epicarpo interno y mesocarpo. Se observan cuatro capas de fibroesclereidas del mesocarpo; L: mesocarpo; M: esclereidas del endocarpo en corte transversal. Se observa pared engrosada; $\mathbf{N}$ : detalle de esclereidas de capa media del endocarpo con disposición irregular.

y Dantas 2006; Biurrun et al. 2007; Scarpa 2012). Asimismo, en este último sitio se reporta la ingesta de frutos hervidos solos o con leche vacuna (Scarpa
2012). Además, estas drupas son aprovechadas por pobladores de otras zonas, como los ranqueles (La Pampa), que las consumen frescas, elaboran bebidas 


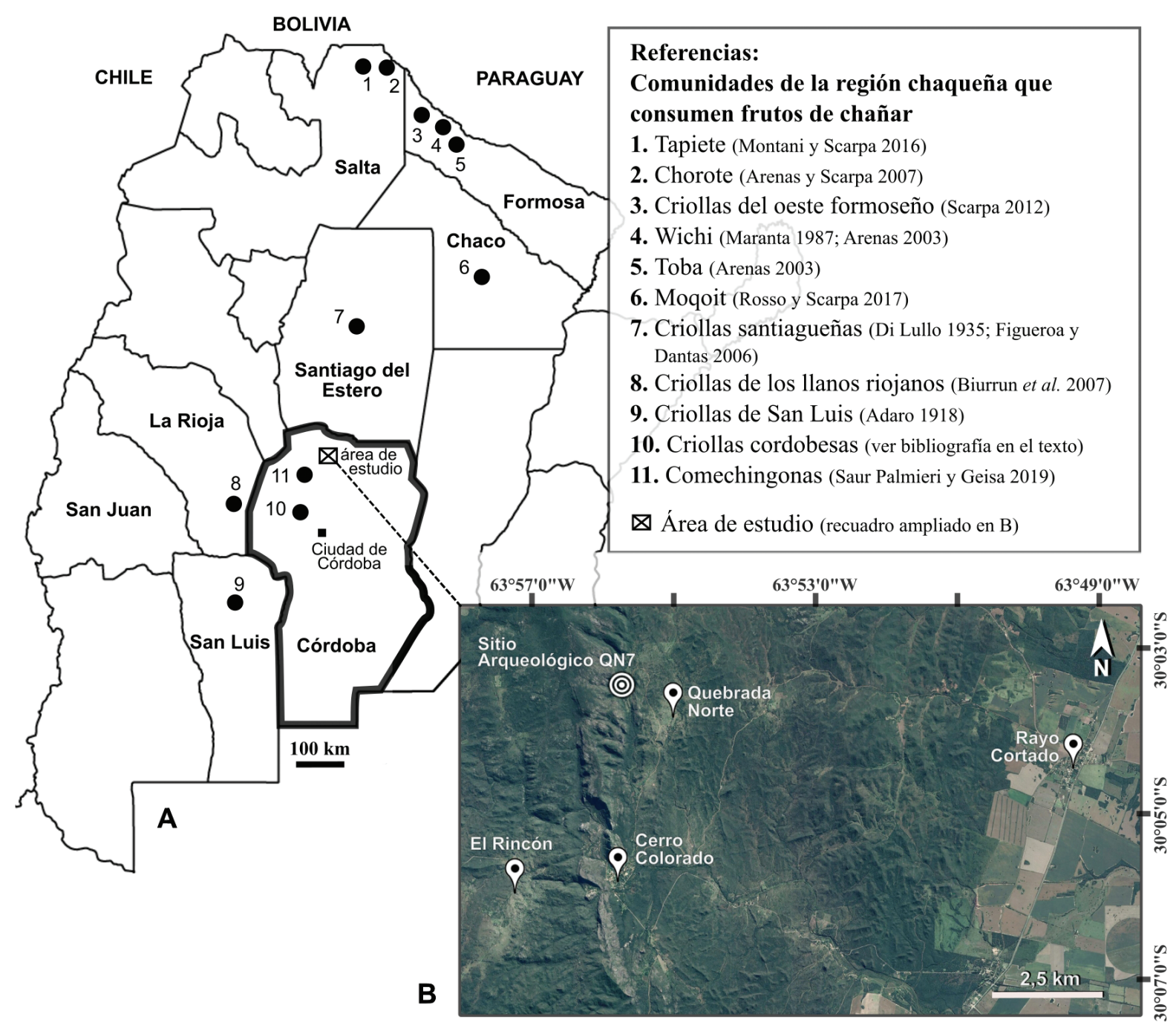

Figura 2. A. Ubicación de las comunidades chaqueñas argentinas que consumen chañar y posición relativa del área de estudio en el noroeste de la provincia de Córdoba. B: Detalle del área de estudio: Cerro Colorado y alrededores, en relación con el sitio arqueológico QN7. Adaptación de imagen satelital (Google Inc., 2017).

refrescantes y fermentadas y emplean sus semillas crudas y cocidas (Steibel 1997). En la misma provincia, los criollos los ingieren como golosina y elaboran arrope (Muiño 2012), mientras que en comunidades sanjuaninas se cita el arrope (Meglioli et al. 2008). En Atacama (Chile) se consume el chañar crudo, en arrope y tostado para elaborar un postre y una bebida denominada "cocho" (Villagrán y Castro 2004).

El arrope constituye un jarabe espeso realizado con la cocción prolongada de frutos, sin el agregado de azúcar. Si bien en el presente estudio se ha trabajado sobre el arrope debido a que su cocción forma parte del cotidiano de los pobladores del área de estudio, se hará referencia a este solo como conjunto de técnicas para procesar los frutos. Esto se debe a que, si bien el término "arrope" proviene del vocablo árabe para denominar el jugo de frutas cocido, no se conoce con claridad cuál ha sido su origen. En este sentido, Scarpa (1999) explicita dos hipótesis: pudo haber surgido a partir de los jarabes medicinales que los árabes introdujeron en España o de la elaboración prehispánica de bebidas en el actual territorio peruano, ya que ambos casos implican el hervor prolongado de frutas.

Algunos documentos históricos, como las memorias de Schmidel, militar alemán del siglo XVI que recorrió las cercanías de Asunción del Paraguay, apoyarían la hipótesis del origen americano del arrope. Él escribió que los aborígenes piembas (payaguás) realizaban "miel" de las vainas de algarroba: "los piembas no tienen más comida que pescado y carne, iten cuerno de cabra, o algorabo o pan de San Juan; de estos cuernos de cabra (vainas de algarroba) hacen ellos miel que comen con el pescado" (Schmidel 2019 [1567]: 169). Ya que el término "miel" es sinónimo de "arrope" (Scarpa 1999), se podría suponer la realización de 
un producto similar entre los originarios de esta región americana. Asimismo, Gerónimo de Bibar, en sus crónicas sobre Chile, relata la cocción de los frutos de molle (probablemente Schinus molle L.) para elaborar una "miel" similar al arrope:

Hay [...] otro árbol que se dice molle y no es muy grande; tiene la hoja como granado y lleva un fruto tan grande como granos de pimienta; lleva muy gran cantidad. De esta fruta se hace un brebaje gustoso, cociendo estos granos en agua muy bien se hace miel que queda a manera de arrope (Bibar 1966 [1558]: 132).

Sin embargo, ningún antecedente bibliográfico da cuenta de su preparación por parte de las etnias originarias del Gran Chaco en los registros etnobotánicos existentes y que datan de los últimos 55 años (ver Scarpa 2009).

Muchas interpretaciones realizadas sobre los pobladores prehispánicos del sector serrano cordobés parecen haber sido basadas en los datos obtenidos desde el noroeste argentino, sin existir correspondencia con el registro arqueológico (Pastor et al. 2012; Recalde y López 2017). Es por ello que se recalca la necesidad de menor distancia entre poblado actual y sitio arqueológico. Además, debido a los últimos hallazgos arqueobotánicos en el norte cordobés (Recalde y López 2017; López 2018) que han dado cuenta de una gran variabilidad de recursos alimenticios consumidos por los habitantes del período Prehispánico tardío (PPT, 1500-300 AP) y de la posible diferenciación en la selección de especies entre subregiones dentro de la gran región serrana cordobesa-, se procedió a reconocer el uso actual de frutos silvestres en esta subregión septentrional.

La evidencia de macrorrestos de chañar arqueológicos obtenida hasta el momento en Córdoba corresponde a dos fragmentos de endocarpo carbonizados recuperados de Arroyo Tala Cañada 1 (valle de Salsacate-subregión oeste) y Quebrada Norte 7 (Sierras del Norte-subregión homónima), sitios ocupados durante el PPT (López 2015, 2018; Recalde y López 2017). También se hallaron microrrestos en tiestos cerámicos de sitios del PPT (Guasapampa, Punilla y Pampa de Achala) (López 2015).

Asimismo, en Córdoba existe una vasta documentación que alude al uso alimenticio actual de chañar en poblaciones criollas (Arias Toledo et al. 2007a y b; Trillo et al. 2014; Martínez et al. 2016; Sánchez 2018; Saur Palmieri et al. 2018; Fernández y Martínez 2019) y en comunidades recientemente reidentificadas como pertenecientes al pueblo comechingón (Saur Palmieri y Geisa 2019). Si bien los pobladores de esta provincia no basan su alimentación en recursos silvestres (Arias Toledo et al. 2007a), estos sí forman parte de su cotidianeidad. El arrope de chañar constituye la forma de consumo más conocida y realizada con estos frutos (Saur Palmieri et al. 2018). A esta información se añaden los documentos españoles de los primeros años de la conquista, que dan cuenta -aunque indirectamente- de su consumo por parte de los grupos nativos (Archivo Histórico de Córdoba, legajo 72, exp.2, f.185v, 19, 1639; Relación Anónima 1572, en Bixio y Berberián 1984).

Ante lo expuesto, resulta imperioso desentrañar las formas de utilización presentes y antiguas, para así evaluar cambios y continuidades en las modalidades de ingesta de dicho vegetal nativo de Córdoba. Es por tal que el objetivo de este trabajo es identificar atributos macro y micromorfológicos de carácter diagnóstico en los frutos actuales recolectados y de cada etapa de procesamiento a las cuales son sometidos con el fin de reconocer aqueIlos rasgos en los carporrestos recuperados arqueológicamente y así descifrar las posibles prácticas realizadas en el pasado. Es importante recalcar que, aunque una práctica presente no refleje exactamente una pretérita, se puede apelar al principio del razonamiento analógico, de acuerdo con el cual la fuente (práctica actual) no es precisamente igual al sujeto (práctica pasada), pero su similitud permite estructurar lógicas de argumentación que posibilitan a la segunda ser interpretada en base a la primera (Politis 2015). Asimismo, es imperioso aclarar que, si bien este trabajo tomará como referencia de cocción de frutos al arrope -producto cuyo origen no se ha comprobado sea americano-, se considerarán las etapas de procesamiento individualmente.

\section{MATERIALES Y MÉTODOS}

\section{Área de estudio}

Comprende la población de Cerro Colorado, situada en el norte de la provincia de Córdoba (Depto. Río Seco) debido a la cercanía al sitio arqueológico Quebrada Norte 7 (Recalde y López 2017). También se abarcaron las localidades aledañas: El Rincón, Rayo Cortado y Quebrada Norte (Figura 2). La zona pertenece al Chaco Serrano de la Provincia Fitogeográfica Chaqueña (Cabrera 1976). Aquí, las precipitaciones disminuyen del este del 
sistema serrano al oeste (de 600 a menos de 500 $\mathrm{mm}$ anuales) y la temperatura media anual aumenta en sentido contrario, de $18,6{ }^{\circ} \mathrm{C}$ al este a $19,9{ }^{\circ} \mathrm{C}$ al oeste. La región comprende el ecotono entre el bosque serrano (oeste) y el bosque chaqueño de llanura (al este). El primero se caracteriza por la presencia de Lithraea molleoides (Vell.) Engl., Myrcianthes cisplatensis (Cambess.) O. Berg y Condalia montana A. Cast., entre otras; y el segundo, por Aspidosperma quebracho-blanco Schltdl., Stetsonia coryne (SalmDyck) Britton \& Rose, mistol, chañar, entre otras (Cabido y Zak 1999).

\section{Contexto sociocultural}

Los pobladores rurales actuales del noroeste de Córdoba se consideran a sí mismos "criollos"1 (Trillo et al. 2014). Esta noción comprende a pequeños productores extensivos de ganado caprino, y bovino en menor medida, que realizan otras actividades tradicionales, como producción agrícola diversificada y en pequeña escala, colecta de frutos de monte, hilados, etc. Obtienen un ingreso monetario extra de la venta de bienes y servicios, empleo en instituciones públicas, etc. Asimismo, desde mediados de la década de 1990, la expansión de la agricultura industrial compromete dicho modo de vida campesino en íntima relación con el bosque nativo (Silvetti y Cáceres 1998; Cáceres 2014).

\section{Etnobotánica-etnoarqueológica}

Para conocer las formas de consumo de chañar en el norte cordobés se utilizó información de un estudio previo en el cual fueron entrevistados 11 pobladores de Cerro Colorado, El Rincón, Rayo Cortado y Quebrada Norte (ver Saur Palmieri et al. 2018). La concordancia entre los resultados de las entrevistas permitió realizar la observación participante (Guber 1991) de la elaboración de arrope en diciembre de 2015 con una de las colaboradoras de Cerro Colorado. Así, se obtuvo la cadena operativa de dicho procesamiento, la serie de pasos que conducen a la transformación de la materia prima en un producto (Cresswell 2010). En este contexto, se considera "residuo" al material vegetal resultante de una etapa de la cadena operativa que es desechado, que sale de su curso por no cumplir ningún rol en las siguientes fases. Se hablará de "frutos procesados" en referencia a las estructuras carpológicas a las que se les han aplicado prácticas poscolecta con el fin de elaborar un determinado producto, como consecuencia de las cuales pueden adquirir características particulares. A diferencia de los residuos, los frutos procesados no se descartan, sino que son parte de la cadena operativa. Además, no necesariamente se destinan a la preparación de otro alimento (Capparelli 2008).

En este trabajo se realizó el registro de las distintas prácticas observadas y categorizadas en precolecta, colecta, poscolecta y consumo como proponen Capparelli y Lema (2010); sin embargo, solo se focalizará en las dos últimas fases, correspondientes a los pasos de transformación del recurso y su utilización final (la ingesta), debido a que las otras etapas no dejan evidencias materiales posibles de registrar a nivel arqueológico (Saur Palmieri et al. 2018).

Para conocer la morfoanatomía de los frutos, estos fueron hervidos 60 minutos; se utilizó la técnica de peeling y se realizaron cortes histológicos (D'Ambrogio de Argüeso 1986). Los preparados se montaron tanto en agua destilada como en aceite de inmersión. Para caracterizar los tejidos, los cortes macroscópicos fueron observados con lupa manual (U500X-DigitalMicroscope-CoolingTech), y los preparados, en microscopio estereoscópico de incidencia y de transparencia (50-200 magnificaciones, Leica). Se utilizaron para este fin frutos colectados de Cerro Colorado (diciembre de 2015), secados a temperatura ambiente y conservados con naftalina.

De la observación participante se obtuvieron muestras de los frutos procesados y residuos luego de cada una de las etapas de la cadena operativa. El producto final, el arrope, al corresponder a un jarabe sin restos visibles macroscópicamente, no fue analizado en el presente trabajo debido a que excede al objetivo propuesto.

Dado que el consumo de chañar también se efectúa por ingesta directa, sin involucrar procesamiento alguno (a excepción del ocasional lavado), y que los endocarpos son desechados, se colectaron los residuos generados por este "consumo directo", para su posterior análisis.

Las muestras constituidas por material actual obtenidas de la elaboración de arrope y del consumo directo fueron secadas a temperatura ambiente y conservadas en naftalina. En forma posterior se las caracterizó macroscópicamente para definir las particularidades cualicuantitativas de los frutos sin procesamiento y los indicadores que sirvan para reconocer prácticas de procesamiento en macrorestos arqueológicos (Capparelli 2008; López et al. 
2011). Se consideraron la presencia/ausencia de los tejidos carpológicos, su estado de preservación y cambios en apariencia (Capparelli y Lema 2011). En los endocarpos, se registró el porcentaje de superficie cubierta por epicarpo y mesocarpo. Con estos caracteres, se establecieron categorías morfológicas cualitativas de las estructuras o partes de estructuras que se consideró pudieran ser diagnósticas para establecer el tipo de práctica poscolecta en el cual las drupas estuvieron involucradas. Se obtuvo el porcentaje de cada categoría con respecto a la cantidad de estructuras y a la masa inicial de cada muestra. Se empleó balanza analítica PK352-Denver Instrument (precisión = 0,01 g). En endocarpos y frutos enteros, también se contabilizaron las unidades presentes en cada categoría para obtener su proporción con relación al total de la muestra.

\section{RESULTADOS}

\section{Morfología del fruto fresco de chañar}

El chañar presenta una drupa, es decir, consta de epicarpo, mesocarpo y endocarpo, que los pobladores actuales denominan "cascarita" o "piel", "pulpa" y "semilla", respectivamente. El epicarpo consiste en cinco capas de células que pueden observarse en el corte transversal del fruto (Figura 1G, H). La epidermis, capa más externa, está constituida por células tabulares y cutícula gruesa (Figura $1 \mathrm{H}$ ). En vista superficial, sus células poseen contorno poligonal y se visualizan estomas aislados. Se percibieron pelos, como mencionan Flores y Vignale (2010) para esta especie (Figura 1E, F). Por debajo de la epidermis se encuentra la hipodermis, de cuatro capas de células colenquimáticas alargadas y con espesamientos en las membranas tangenciales (Figura 1H, K).

El mesocarpo está formado principalmente por múltiples capas de células parenquimáticas grandes $(\approx 50 \mu \mathrm{m})$, en forma de saco y con contenido ocráceo. ${ }^{2}$ Posee cuatro hileras de fibroesclereidas (sensu Esau 1985) que forman una capa continua bajo la hipodermis y, a escala macroscópica, se visualiza blancuzca-translúcida (Figura 1G, H, K, L). Asimismo, se observan miembros de vaso espiralados en lo que posiblemente sean los haces vasculares del fruto (Figura 1L).

El endocarpo -grueso y duro- posee dos capas de esclereidas de paredes engrosadas e incoloras y una capa interna de células parenquimáticas. En la capa externa, las esclereidas se encuentran con su eje longitudinal perpendicular a la superficie del endocarpo, mientras que en la capa media están dispuestas ordenadamente, de forma compacta, hacia el exterior e irregularmente hacia el interior (Figura 1I, J, M, N). La capa interna del endocarpo, que recubre los surcos transversales que se aprecian a simple vista, es blancuzca, hialina y posee brillo sedoso. Las células parenquimáticas ${ }^{3}$ que la forman poseen paredes delgadas y aspecto etéreo y translúcido, observable con lupa (Figura 1I). ${ }^{4}$

\section{Rasgos de procesamiento}

Durante las diferentes etapas de cocción de los frutos para la elaboración de arrope (Figura 3), se observaron cambios y pérdida de estructuras.
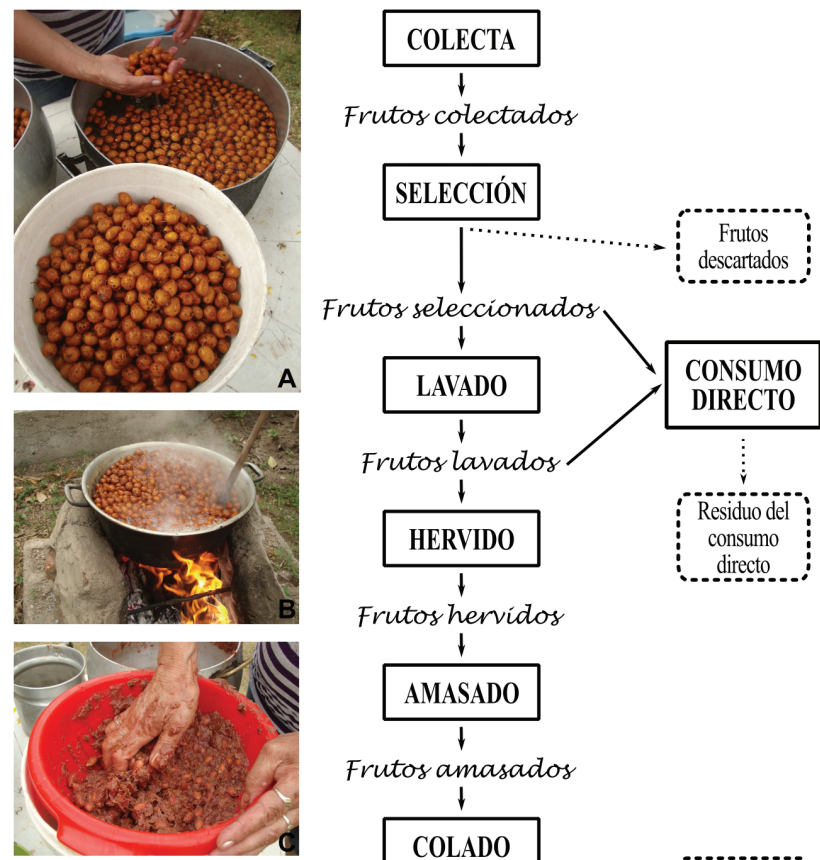

Frutos amasados

$\downarrow$
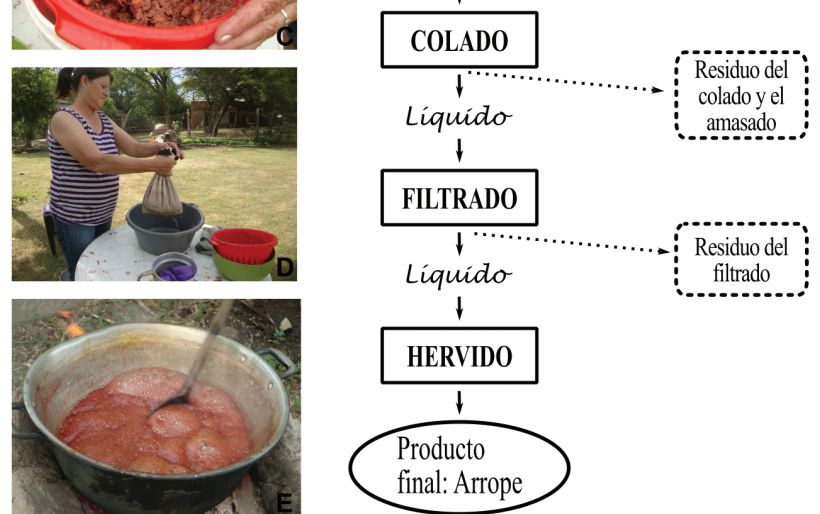

Figura 3. Cadena operativa de arrope de chañar registrada durante la observación participante en Cerro Colorado. Se incluye también la ingesta de los frutos sin procesamiento. A: lavado; B: hervido inicial de los frutos; C: chaguado y colado simultáneos; D: filtrado; E: hervido del líquido. 
En el lavado de las drupas -que consiste en colocarlas en un recipiente con abundante agua para quitar las impurezas adheridas-, estas permanecen enteras y mantienen el pedicelo (Figura 3A). Su epicarpo conserva el brillo y color original; puede presentar hundimientos leves, pero en general permanece liso y continuo, sin fracturas. No obstante, se pierden pelos tectores, lo que puede atribuirse a la fricción provocada entre los propios frutos al ser lavados. Los pedicelos no se pierden a menos que se retiren intencionalmente, tal como algunos entrevistados indicaron.

Por su parte, el hervido (Figura 3B) mantiene los frutos enteros, pero ablanda el epicarpo, y el $30 \%$ pierde su pedicelo. Adquieren coloración marrón, mantienen el brillo y presentan el epicarpo notablemente arrugado y con hundimientos. En vista superficial, la epidermis exhibe numerosos pliegues (que son los que brindan al epicarpo un aspecto rugoso) (Figura 4A), a lo largo de los cuales se encuentra separada de la subyacente hipodermis (Figura 4C, D). En corte transversal se observa que cutícula y células epidérmicas adquieren aspecto homogéneo como si estuvieran amalgamadas, luego del sometimiento a la temperatura alta del hervor (Figura 4D). El $31,6 \%$ de los frutos hervidos presenta fracturas en epicarpo apreciables a simple vista, por lo general, en sentido longitudinal respecto del eje mayor del fruto. La epidermis puede encontrarse fracturada $y$, muchas veces, también la hipodermis. En ambos casos, la primera presenta enrollamientos en sus bordes. Cuando la hipodermis queda expuesta, se puede apreciar como una capa homogénea y opaca (Figura 4B).

Durante el amasado (o "chaguado"), se rompe la pared del fruto con las manos y se desmenuza
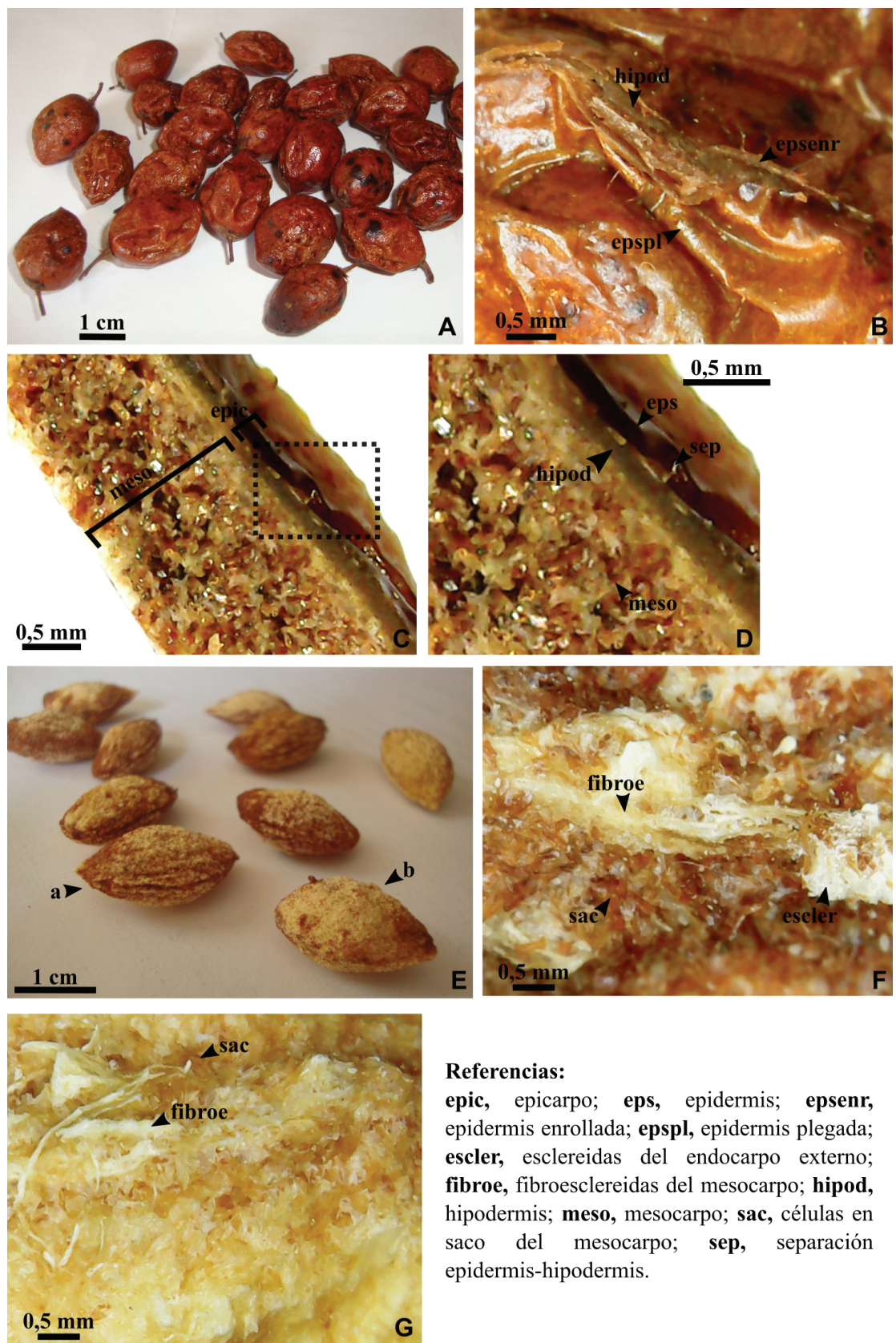

\section{Referencias:}

epic, epicarpo; eps, epidermis; epsenr, epidermis enrollada; epspl, epidermis plegada; escler, esclereidas del endocarpo externo; fibroe, fibroesclereidas del mesocarpo; hipod, hipodermis; meso, mesocarpo; sac, células en saco del mesocarpo; sep, separación epidermis-hipodermis.

Figura 4. Frutos de chañar hervidos y residuo del consumo directo. A: vista general de frutos hervidos; $\mathbf{B}$ : detalle de la fractura de epicarpo y plegamiento de epidermis de fruto hervido. Se observa hipodermis por debajo de la epidermis enrollada en el borde de la fractura; C: corte transversal de fruto hervido; D: detalle del recuadro punteado en C, donde se observa separación de epidermis e hipodermis; E: vista general. Se observan endocarpos con distinto porcentaje de mesocarpo adherido: a) endocarpos con más del $50 \%$ de mesocarpo adherido y pátina oscura entre las rugosidades del endocarpo; b) endocarpos con menos del $50 \%$ de mesocarpo depositado principalmente en áreas distales; F: superficie de endocarpo de la categoría con más del $50 \%$ de mesocarpo, región de la sutura carpelar. Detalle de haces de fibroesclereidas dispuestas con su eje mayor paralelo a la línea de sutura; G: superficie de endocarpo de la categoría con menos del $50 \%$ de mesocarpo, región de la sutura carpelar. Se observan escasas células en saco del mesocarpo y haces de fibroesclereidas fragmentados y poco notorios a lo largo de la línea de sutura. 
el mesocarpo (Figura 3C). Junto con el colado procedimiento que supone refregar el material por el colador, durante el cual se puede agregar agua para facilitar la tarea-, generan un único residuo conformado por epicarpos sueltos, frutos enteros, pedicelos y endocarpos (Figura 5A-F). Los epicarpos sueltos representan el $16,4 \pm 6,8 \%$ de la masa total del residuo y se encuentran en forma de hojuelas. Presentan las mismas características que el epicarpo de frutos hervidos (Figura 5A, G, H).

Por su parte, los frutos enteros encontrados en este residuo -es decir, que presentaron una alta proporción de los tejidos en su posición original luego del procesamientono experimentaron cambios sustanciales y representan un $4,0 \pm 2,9 \%$ de la masa total del residuo. Pueden aparecer globosos (Figura 5B) o notablemente aplastados (Figura 5C), pero siempre se diferencian de los hervidos por exhibir fragmentos de mesocarpo de otros frutos adheridos a su superficie (Figura 5I). Asimismo, todos tienen el epicarpo fracturado, con las características citadas para la fractura de los frutos hervidos. En los aplastados, las fracturas fueron más grandes y presentaron mayor exposición de mesocarpo (Figura 5C). Se encontraron pedicelos sueltos y adheridos al epicarpo, que representan un $2,7 \pm 0,6 \%$ de la masa del residuo (Figura 5D).

El $76,9 \pm 18,4 \%$ de la masa del resultantes del filtrado. residuo del chaguado-colado está representado por endocarpos, todos enteros, sin fracturas. En su superficie se encuentran adheridos fragmentos del epicarpo y del mesocarpo (probablemente procedentes de varios frutos), en distinta proporción y con distinto grado de preservación, dependiendo de cuán amasado y frotado con el colador estuviera el endocarpo (Figura 5E, F, J, K y Tabla 1 del Material Complementario). Los fragmentos de epicarpo muestran epidermis e hipodermis, o solo hipodermis. El mesocarpo se observa poroso a simple vista, y sus haces de fibroesclereidas se encuentran principalmente en forma de pequeños fragmentos
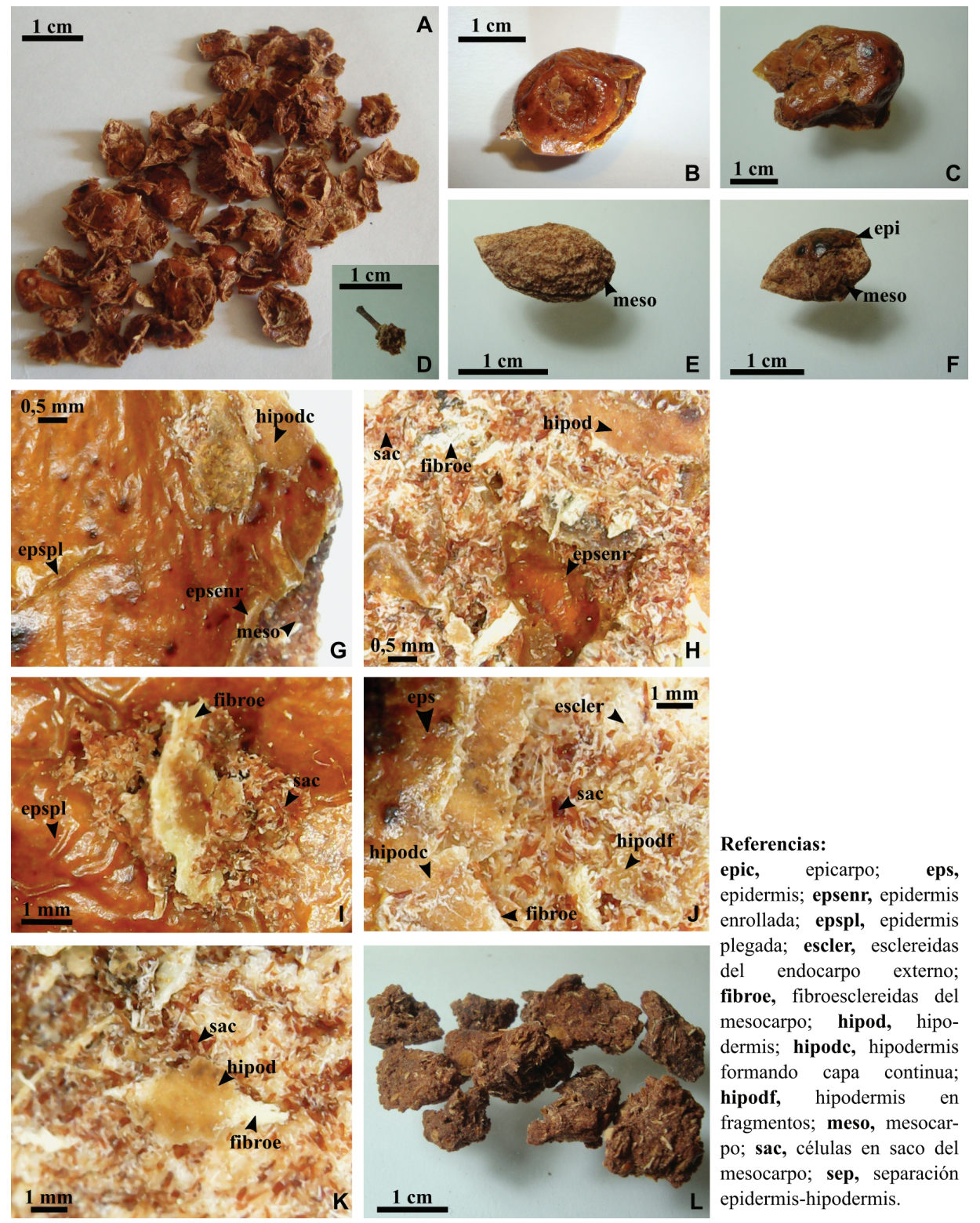

Referencias:

epic, epicarpo; eps, epidermis; epsenr, epidermis enrollada; epspl, epidermis plegada; escler, esclereidas del endocarpo externo; fibroe, fibroesclereidas del mesocarpo; hipod, hipodermis; hipodc, hipodermis formando capa continua; hipodf, hipodermis en fragmentos; meso, mesocarpo; sac, células en saco del mesocarpo; sep, separación epidermis-hipodermis.

Figura 5. Residuo de amasado ("chaguado") y colado (A-J) y del filtrado (I). A: hojuelas de epicarpo; B: "fruto entero globoso"; C: "fruto entero aplastado"; D: pedicelo; E: endocarpo. Se observa mesocarpo poroso entre las rugosidades de superficie. F: endocarpo. Se observa epicarpo y mesocarpo poroso; G: hojuela de epicarpo, lado exterior; H: hojuela de epicarpo, lado interior; I: superficie de fruto entero. Se observan adherencias de elementos mesocárpicos (células en saco) posiblemente de otro fruto; J y K: superficie de endocarpo; L: conglomerados 
mezclados con las células en saco. También se destacan porciones de haces de fibroesclereidas con hipodermis (Figura $5 \mathrm{~J}, \mathrm{~K}$ ).

Con relación al filtrado -realizado a través de una tela (Figura 3D) y cuyo fin es purificar el líquido dulce para luego espesarlo por hervido-, este presenta como residuo una masa compacta de mesocarpo y epicarpo (Figura 5l).

Los distintos instrumentos empleados en la realización de arrope registrados durante la observación participante se listan en la Tabla 2 (Material Complementario) y se compara con el instrumental utilizado en el pasado, como es recordado por los entrevistados.

\section{Rasgos de consumo directo}

Esta forma de ingesta solo implica el lavado previo de los frutos y consiste en ingerir únicamente el mesocarpo dulce y blando, deshaciendo todo el fruto dentro de la boca. Así, el residuo de esta práctica está constituido únicamente por endocarpos enteros, sin fracturas ni trazas de epicarpo. Presentan remanentes de mesocarpo adherido, que se pueden distinguir en dos grupos (Figura 4E). El primero ( $48 \%$ de los endocarpos) presentó más del $50 \%$ de mesocarpo en forma de pátina oscurecida depositada a lo largo de la línea de sutura y entre las rugosidades de la superficie. Exhibía, asimismo, a lo largo de la sutura carpelar, haces de fibroesclereidas bien notables (Figura 4F). El segundo grupo (el $52 \%$ restante) poseía menos del $50 \%$ de mesocarpo adherido. Este tejido adoptó el aspecto de pátina de color claro, distribuida principalmente en sectores distales del endocarpo. A diferencia de la primera categoría, los haces de fibroesclereidas en la sutura carpelar estaban fragmentados (Figura 4G).

En la Tabla 3 (Material Complementario) se puede observar el resumen de los caracteres diagnóstico aquí expuestos.

\section{DISCUSIÓN Y CONCLUSIÓN}

En este trabajo se registraron las prácticas realizadas en la actualidad en la localidad de Cerro Colorado y alrededores sobre los frutos de chañar destinados a la ingesta, cuya finalidad es poseer una base de información etnográfica para la interpretación de las prácticas pretéritas poscolecta en el material arqueológico. De esta manera, se presentaron dos opciones de ingesta, con procesamiento y sin él, y se describieron los atributos fijados en las estructuras carpológicas por cada una de las técnicas empleadas. Se ha focalizado en la cocción de arrope debido a que es un producto ampliamente manufacturado en la actualidad en la zona, lo que permite así presenciar su realización, registrar cada una de sus etapas y tomar muestras para su análisis. No obstante, su surgimiento sigue siendo una incógnita; si bien en este trabajo se presentan algunas evidencias documentales que podrían sustentar su origen y desarrollo independiente tanto en América como en el Viejo Mundo, a pesar de su nombre de raíz árabe.

El análisis macromorfológico de las drupas sometidas al procesamiento culinario se realizó para entender los cambios sufridos por las distintas partes de los frutos durante cada etapa de la cadena operativa: hervido, amasado, colado. La práctica que no imprime características peculiares a la estructura de los frutos la representa el lavado, porque su único fin es limpiar el epicarpo. No obstante, deberían evaluarse otros procesamientos y la existencia de condiciones tafonómicas que podrían producir la pérdida de pelos tectores, lo cual indica, hasta el momento, la selección y lavado de los frutos.

El hervido ablanda el epicarpo, debilita la epidermis, se pliega y fractura, y el aspecto rugoso del tejido puede reconocerse en fragmentos de epicarpo de los subsiguientes pasos del procesamiento. Como los frutos frescos, los secos y los lavados tienen epicarpo liso; la presencia de pliegues representa un rasgo ineludible del hervido. Otro rasgo distintivo lo constituyeron los hundimientos de pared.

El amasado y el frotado del material con el colador contribuyen a triturar los tejidos carpológicos, que se encuentran en distinto nivel de degradación en el residuo de esta fase de elaboración. Por esta razón, se encontraron abundantes endocarpos con adherencias de epicarpo y mesocarpo, fragmentos de epicarpos sueltos, algunos frutos enteros que no fueron segmentados en sus distintas partes pero que presentaron restos de epicarpo y mesocarpo adheridos al exterior, y pedicelos que se desprendieron del epicarpo. Los endocarpos, duros, con baja proporción de su superficie cubierta por epicarpo, constituyen la mayor parte de este desecho, lo cual indica la efectiva trituración de las estructuras carpológicas blandas, factibles de ser degradadas manualmente. Es decir, los trozos de epicarpo y mesocarpo que alcanzaron un tamaño menor que el del orificio 
del colador pasan a formar parte del líquido espeso que será filtrado, y las porciones de mayor tamaño son desechadas (tal es el caso de frutos enteros, fragmentos grandes de epicarpo y endocarpos).

Con el filtrado se logra la separación total de los azúcares diluidos en el líquido, de los fragmentos de tejido que quedan retenidos en la trama de la tela utilizada. Los conglomerados se forman al estrujar el contenido del filtro y quedan compactos cuando se seca el material.

La ingesta de las drupas como fruta fresca genera un residuo formado únicamente por endocarpos, sin epicarpo adherido y con fibroesclereidas en la sutura carpelar que diagnostican su procedencia. La disimilitud entre los dos grupos de endocarpos observados podría ser atribuida a "distintos patrones de consumo", como la diferente cantidad de tiempo que estuvo el endocarpo en boca del individuo, o si se mordió con los dientes o no. No obstante, queda por cuantificar la influencia de estas variables en las características de los carozos residuos de esta práctica. Por otra parte, a pesar de que el residuo del chaguado también está constituido principalmente por endocarpos, estos están acompañados de otras partes del fruto $y$, a su vez, poseen otros rasgos que son distintivos (ver más arriba).

Ante lo expuesto, se pudo constatar que el hervido, la trituración manual o el consumo directo del chañar no generan la ruptura del endocarpo. Por consiguiente, se podría adelantar que los endocarpos de chañar arqueológicos hallados hasta el momento en Córdoba, que se encuentran fragmentados, no se han producido por ninguna de estas prácticas. No obstante, deberían evaluarse otras técnicas, como el machacado en mortero y tostado, procesos respecto de los cuales -aunque actualmente no se realizan con estos frutos en el área de estudio- hay registros etnográficos para otras zonas (e.g., Arenas 2003; Villagrán y Castro 2004). Debido a que los carporrestos arqueológicos se presentan carbonizados, es necesaria la generación de réplicas experimentales con la misma exposición a altas temperaturas para poder evaluar cambios en los rasgos diagnósticos de las estructuras carpológicas luego de cada técnica y las probabilidades de preservación en el registro arqueobotánico luego del tratamiento con calor (Capparelli 2011). Así, se podrán a futuro discriminar lo más posible los efectos naturales de posdepositación de las prácticas pretéritas en las que las drupas estuvieron involucradas.

En suma, se caracterizaron las estructuras y partes de estructuras de frutos de chañar procesados y residuos de la cadena operativa culinaria más realizada en Cerro Colorado, y del consumo directo. Se determinó que cada una de las fases genera un ensamble particular de restos (usados en fases posteriores o descartados) y a su vez, imprime rasgos notorios y típicos en los tejidos que permiten distinguir en seco qué prácticas fueron efectuadas sobre ellos.

\section{Agradecimientos}

Agradecemos a Ángela y todas las personas que colaboraron gentilmente con este trabajo. A las Dras. A. Capparelli y D. Vignale. A las y los evaluadores que, con sus aportes, contribuyeron a mejorar esta presentación. El presente trabajo se realizó en el marco de una Beca del CIN y del proyecto "Percepción, manejo y uso de unidades de paisaje en zonas rurales de Córdoba" (Res.313/16-SECYT-UNC).

\section{REFERENCIAS CITADAS}

Adaro, D. S.

1918 Industrias criollas o fitotecnia. Aplicaciones de vegetales indígenas y exóticos. Weiss y Preusche, Buenos Aires.

Arenas, $\mathrm{P}$

2003 Etnografía y alimentación entre los Toba-

Ñachilamole\#ek y Wichí-Lhuku'tas del Chaco Central

(Argentina). Edición del autor, Buenos Aires.

Arenas, P. y G. F. Scarpa

2007 Edible wild plants of the chorote Indians, Gran Chaco, Argentina. Botanical Journal of the Linnean Society 153 (1): 73-85.

Arias Toledo, B., S. Colantonio y L. Galetto 2007a Knowledge and use of edible and medicinal plants in two populations from the Chaco forest, Córdoba Province. Journal of Ethnobiology 27 (2): 218-232.

Arias Toledo, B., L. Galetto y S. Colantonio 2007b Uso de plantas medicinales y alimenticias según características socioculturales en Villa Los Aromos (Córdoba, Argentina). Kurtziana 33: 79-88.

Bibar, G. de 1966 [1558] Crónica y relación copiosa y verdadera de los reynos de Chile. Transcripción paleográfica de I. Leonard. Introducción de G. Feliú-Cruz. Fondo Histórico y Bibliográfico J. T. Medina, Santiago de Chile. http:// www.memoriachilena.gob.cl/602/w3-article-8175.html 
Biurrun, E., L. Galetto, A. M. Anton y N. Biurrun 2007 Plantas silvestres comestibles utilizadas en poblaciones rurales de la provincia de La Rioja (Argentina). Kurtziana 33: 121-240.

Bixio, B. y E. Berberián 1984 Etnohistoria de la región de Potrero de Garay (Córdoba-Argentina). Comechingonia 3: 11-46.

Bompadre, J. M.

2013 Procesos de comunalización contemporánea de pueblos originarios en contextos urbanos y rurales de Córdoba. X Reunión de Antropología del Mercosur. Córdoba.

Cabido, M. y M. Zak 1999 Vegetación del Norte de Córdoba. Secretaría de Agricultura, Ganadería y Recursos Renovables, Córdoba.

Cabrera, A. L. 1976 Regiones fitogeográficas argentinas. En Enciclopedia Argentina de Agricultura y Jardinería, tomo 2, fasc.1, editado por W. F. Kugler, pp. 1-85. ACME, Buenos Aires.

\section{Cáceres D. M.}

2014 Amenazas y desafíos que enfrenta el campesinado en Argentina. ¿Descampesinización o persistencia? En Agricultura Familiar en Latinoamérica. Continuidades, transformaciones y controversias, editado por C. Craviotti, pp. 205-232. Ciccus, Buenos Aires.

Capparelli, A. 2007 Los productos alimenticios derivados de Prosopis chilensis (Mol.) Stuntz y P. flexuosa DC., Fabaceae, en la vida cotidiana de los habitantes del NOA y su paralelismo con el algarrobo europeo. Kurtziana 33 (1): 1-19.

2008 Caracterización cuantitativa de productos intermedios y residuos derivados de alimentos del algarrobo (Prosopis flexuosa y P. chilensis, Fabaceae): aproximación experimental aplicada a restos arqueobotánicos desecados. Darwiniana 46 (2): 175-201.

2011 Elucidating post-harvest practices involved in the processing of algarrobo (Prosopis spp.) for food at El Shincal Inka. Archaeological and Anthropological Sciences 3 (1): 93-112.

Capparelli, A. y V. Lema

2010 Prácticas poscolecta/post-aprovisionamiento de recursos vegetales: una perspectiva paleoetnobotánica integradora aplicada a casos de Argentina. Actas del XVII Congreso Nacional de Arqueología Argentina 3: 1171-1176.
2011. Recognition of post-harvest processing of algarrobo (Prosopis spp.) as food from two sites of Northwestern Argentina: an ethnobotanical and experimental approach for desiccated macroremains. Archaeological and Anthropology Sciences 3: 71-92.

Capparelli, A. y L. Prates

2015 Explotación de frutos de algarrobo (Prosopis spp.) por grupos cazadores recolectores del noreste de Patagonia. Chungara 47 (4): 549-563.

Capparelli, A., S. M. Valamoti y M. M. Wollstonecroft 2011 After the harvest: investigating the role of food processing in past human societies. Archaeological and Anthropology Sciences 3: 1-5.

Cresswell, $\mathrm{R}$. 2010 Techniques et culture. Les bases d'un programme de travail. Techniques \& Culture 54-55 (1): 20-45.

D'Ambrogio de Argüeso, A. 1986 Manual de técnicas en histología vegetal. Hemisferio Sur, Buenos Aires.

Demaio, P., U. O. Karlin y M. Medina 2015 Árboles nativos de Argentina: Centro y Cuyo. Ecoval, Córdoba.

Di Lullo, O.

1935 La alimentación popular de Santiago del Estero. El Liberal, Santiago del Estero.

Esau, K.

1985 Anatomía vegetal. Omega, Barcelona.

Esteve, M.

2009 "Tierra y agua para poder producir y vivir": El Movimiento Campesino Cordobés. Theomai 20: 186-200.

Fernández, A. y G. J. Martínez

2019 Las plantas en la alimentación de pobladores rurales de los ambientes serranos de La Calera (Dpto. Colón, Córdoba, Argentina). Una perspectiva etnobotánica diacrónica. Bonplandia 28 (1): 43-69. doi:10.30972/bon.2813573

Figueroa, G. G. y M. Dantas 2006 Recolección, procesamiento y consumo de frutos silvestres en el noroeste semiárido argentino. Casos actuales con implicancias arqueológicas. Zaranda de Ideas 2: 35-50.

Flores, E. N. y N. D. Vignale

2010 Caracterización micrográfica de órganos vegetativos y reproductivos de interés etnobotánico de Geoffroea decorticans (Gill. ex Hook. et Arn.) Burkart (Fabaceae). En Tradiciones y transformaciones en etnobotánica, editado por M. L. Pochettino, A. H. Ladio y P. M. Arenas, pp. 330-335. CYTED/RISAPRET, Jujuy. 
Guber, R.

1991 El salvaje metropolitano. Legasa, Buenos Aires.

Hillman, G. C.

1984. Interpretation of archaeological plant remains: The application of ethnographic models from Turkey. En Plants and Ancient Man. Studies in palaeoethnobotany, editado por W. Van Zeist y W. A. Caspary, pp. 1-41. A. Balkema, Rotterdam.

Hillman, G. C. y M. Davis

1990 Measured domestication rates in wild wheats and barley under primitive cultivation, and their archaeological implications. Journal of World Prehistory 4 (2): 157-222.

Lema, V. S.

2009 Criterios de selección en los procesos de manipulación vegetal: el aporte de la etnobotánica a la interpretación de restos arqueobotánicos de Cucurbita sp. Darwiniana 47 (1): 35-55.

López, M. L.

2015 La cocina como medio para la reproducción social de los grupos prehispánicos de las sierras de Córdoba. En Condiciones de posibilidad de la reproducción social en sociedades prehispánicas y coloniales tempranas en las Sierras Pampeanas (República Argentina), editado por J. Salazar, pp. 177-212. Centro de Estudios Históricos C. S. A. Segreti, Córdoba.

2018. Archaeobotany in Central Argentina. Macro and microremains at several archaeological sites from early Late Holocene to early Colonial Times (3000-250 BP). Vegetation History and Archaeobotany 27 (1): 219-228. doi:10.1007/s00334-017-0627-x

López, M. L., A. Capparelli y A. Nielsen

2011 Traditional post-harvest processing to make quinoa grains (Chenopodium quinoa var. quinoa) apt for consumption in Northern Lipez (Potosí, Bolivia): ethnoarchaeological and archaeobotanical analyses. Archaeological and Anthropological Science 3 (1): 49-70.

Maestri, D. M., R. H. Fortunato, J. A. Greppi y A. L. Lamarque

2001 Compositional studies of seeds and fruits from two varieties of Geoffroea decorticans. Journal of Food Composition and Analysis 14 (6): 585-590.

Maranta, A.

1987 Los recursos vegetales alimenticios de la etnia Mataco del Chaco Centro Occidental. Parodiana 5 (1): 161-237.

Margaritis, E. y M. Jones

2006 Beyond cereals: crop processing and Vitis vinifera
L. Ethnography, experiment and charred grape remains from Hellenistic Greece. Journal of Archaeological Science 33 (6): 784-805.

Martínez, G., C. Romero, C. Pen, M. Villar y P. Durando 2016 Etnobotánica Participativa en escuelas rurales de la Comuna Paso Viejo (Departamento Cruz del Eje, Córdoba, Argentina). Bonplandia 25 (2): 145-162.

Meglioli, C., C. Vega Riveros, P. Meglioli, U.O. Karlin y M. Bedini

2008 Sabores de nuestros pueblos. Uso de los recursos naturales en la alimentación, y comidas tradicionales en las comunidades del Norte de Valle Fértil, San Juan. Universidad Nacional de San Juan, San Juan.

Montani, M. C. y G. F. Scarpa

2016 Recursos vegetales y prácticas alimentarias entre indígenas tapiete del noreste de la provincia de Salta, Argentina. Darwiniana, nueva serie 4 (1): 12-30.

Muiño, W. A.

2012 Estudio etnobotánico de plantas usadas en la alimentación de los campesinos del noroeste de La Pampa Argentina. Chungara 44 (3): 389-400.

Orrabalis, C., H. Gorostegui, E. Calandri y C. Guzmán 2014 Caracterización química y evaluación sensorial de dulces novedosos elaborados con frutos de Geoffroea decorticans. Phyton 83 (1): 117-125.

Pastor, S., M. Medina, A. Recalde, L. López y E. Berberián 2012 Arqueología de la región montañosa central de Argentina. Avances en el conocimiento de la Historia Prehispánica Tardía. Relaciones de la Sociedad Argentina de Antropología XXXVII (1): 89-112.

Pennington, R. T. y B. Gemeinholzer 2000 Cryptic clades, fruit wall morphology and biology of Andira (Leguminosae: Papilionoideae). Botanical Journal of the Linnean Society 134 (1): 267-286.

Pochettino, M. L. y V. S. Lema 2008 La variable tiempo en la caracterización del conocimiento botánico tradicional. Darwiniana 46: 227-239.

Politis, G. 2015 Reflections on Contemporary Ethnoarchaeology. Pyrenae 46 (1): 41-83.

Recalde, A. y L. López 2017 Las sociedades prehispánicas tardías en la región septentrional del centro de Argentina (Sierras del Norte, Córdoba). Avances a su conocimiento desde los recursos vegetales. Chungara 49 (4): 573-588. doi:10.4067/S0717-73562017005000109 


\section{Rosso, C. N. y G. F. Scarpa}

2017 Etnobotánica de la alimentación entre los indígenas moqoit actuales de la provincia del Chaco (Argentina) y comparación con fuentes históricas de los siglos XVIII y XX. Boletín de la Sociedad Argentina de Botánica 52 (4): 827-840.

Sánchez, A.

2018. Aproximaciones Etnohistóricas de los alimentos Prehispánicos e Hispánicos en la posta Guayascate del Camino Real en Córdoba. Tesis de Grado inédita. Facultad de Ciencias Exactas, Físicas y Naturales, Universidad Nacional de Córdoba, Córdoba.

Saur Palmieri, V y M. G. Geisa

2019 Las plantas comestibles empleadas por las comunidades comechingonas de San Marcos Sierras (Córdoba, Argentina). Boletín de la Sociedad Argentina de Botánica. En prensa.

Saur Palmieri, V., M. L. López y C. Trillo 2018 Aproximaciones etnobotánicas de las especies y prácticas de frutos nativos comestibles de la actualidad. Aportes para la interpretación del pasado prehispánico de Cerro Colorado (Córdoba). Boletín de la Sociedad Argentina de Botánica 53 (1): 115-133.

Scarpa, G. F.

1999. El arrope en el Noroeste Argentino. Ayer una fiesta, hoy un capital. En Los sabores de España y América, editado por A. Garrido-Aranda, pp. 93-139. La Val de Onsera, Huesca.

2009 Wild food plants used by the indigenous peoples of the South American Gran Chaco: A general synopsis and intercultural comparison. Journal of Applied Botany and Food Quality 83: 90-101.

2012. Las plantas en la vida de los criollos del oeste formoseño: medicina, ganadería, alimentación y viviendas tradicionales. Rumbo Sur, Buenos Aires.

Schmidel, U.

2019 [1567] Viaje al Río de La Plata. Traducido por S. Lafone Quevedo. Linkgua, Barcelona.
Silvetti, F. y D. Cáceres

1998 Una perspectiva sociohistórica de las estrategias campesinas del noreste de Córdoba, Argentina. Debate Agrario 28: 103-127.

Steibel, P.

1997 Nombres y usos de las plantas aplicadas por los indios ranqueles de La Pampa (Argentina). Revista de la Facultad de Agronomía (UNLP) 9 (2): 1-40.

Trillo, C., S. Colantonio y L. Galetto

2014 Perceptions and Use of Native Forests in the Arid Chaco of Córdoba, Argentina. Ethnobotany Research \& Applications 12: 497-510.

Villagrán, C. y V. Castro

2004 Ciencia indígena de los Andes del norte de Chile. Universitaria, Santiago de Chile.

Winton, A. L. y K. B. Winton

1935 The structure and composition of foods, vol. 2. J. Wiley \& Sons, Nueva York.

\section{NOTAS}

1.- A pesar de que la "identidad criolla" sigue siendo muy aludida en este territorio, algunas personas han comenzado un proceso de autoidentificación, con identidades tales como "campesino" (Esteve, 2009) y "comechingón" (Bompadre 2013), las cuales no deben desconocerse para la caracterización cabal de las comunidades en estudio.

2.- Estas se corresponden con las células que Winton y Winton (1935) describen para drupas de Rosaceae.

3.- Según la bibliografía, el endocarpo de frutos de leguminosas posee una capa de células parenquimáticas de paredes delgadas, donde se encuentra alojada la semilla. A la madurez, dicho tejido se encoge y deseca, y adopta aspecto de papel, similar a escamas (Pennington y Gemeinholzer 2000). En el presente trabajo, esta capa no se observó claramente al microscopio, por lo que resta confirmar su composición celular en futuros trabajos.

4.- Similares características de epicarpo y endocarpo fueron descriptas en el género Andira, Fabáceas, cuyos frutos también son drupas (Pennington y Gemeinholzer 2000). 